\title{
Severe Periodontitis Is Associated With Diastolic Blood Pressure Elevation in Individuals With Heterozygous Familial Hypercholesterolemia: A Pilot Study
}

Carolina L.Z. Vieira, ${ }^{*}$ Patricia R. Cury,$^{\dagger}$ Marcio H. Miname, ${ }^{*}$ Lilton R. Martinez, * Luiz A. Bortolotto, * Isabela B. Giuliano, ${ }^{\ddagger}$ Raul D. Santos, ${ }^{*}$ and Bruno Caramelli*

Background: This pilot study evaluates the association of severe periodontitis with pulse wave velocity (PWV), carotid artery intima-medial thickness (IMT), and clinical, metabolic, and atherogenic inflammatory markers in 79 subjects with heterozygous familial hypercholesterolemia (hFH). All subjects were free of previous vascular disease manifestations.

Methods: The body mass index (in kilograms per square meter), plasma lipids, glucose, C-reactive protein, and white blood cell counts were evaluated. After full-mouth periodontal examinations, patients were categorized into the severe periodontitis group (SPG) or non-severe periodontitis group (NSPG).

Results: The SPG showed significantly higher values of cholesterol-year scores, triglycerides, glucose, PWV, IMT, and diastolic blood pressure (DBP) $(P \leq 0.05)$ than the NSPG. After adjustment for traditional risk factors for atherosclerosis, only the association between severe periodontitis and DBP (odds ratio: $3.1 ; 95 \% \mathrm{CI}: 1.1$ to $8.5 ; P=0.03$ ) was confirmed.

Conclusion: In individuals with $\mathrm{hFH}$, severe periodontitis was associated with a higher DBP, which suggests that severe periodontitis, itself, may contribute to the increased cardiovascular risk profile in this population. J Periodontol 2011;82:683-688.

\section{KEY WORDS}

Carotid artery diseases; hypercholesterolemia; inflammation; periodontal disease; risk factors.

\footnotetext{
* Heart Institute, Medical School, University of São Paulo, São Paulo, SP, Brazil.

$\dagger$ Department of Periodontics, School of Dentistry, Federal University of Bahia, Salvador, BA, Brazil.

‡ Department of Pediatrics, Federal University of Santa Catarina, Florianópolis, SC, Brazil.
}

$\square$ amilial hypercholesterolemia (FH) is the most common classic autosomal dominant inherited disorder, characterized by mutations in the gene encoding the low-density lipoprotein (LDL) receptor protein. ${ }^{1}$ Although homozygous $\mathrm{FH}$ is a rare genetic disorder, the estimated prevalence of heterozygous $\mathrm{FH}(\mathrm{hFH})$ is $0.2 \%$ (one in 500 individuals) in most populations of the world. ${ }^{2}$

$\mathrm{hFH}$ is characterized by a severely elevated concentration of LDL cholesterol (LDL-C) in plasma starting in early childhood, tendon xanthomas, and a markedly increased risk for premature coronary heart disease (CHD). Adequate control of plasma LDL-C levels can be achieved in most patients with $\mathrm{hFH}$, and to a lesser extent, in patients with homozygous $\mathrm{FH}$, by using combinations of diet, drug therapy, and selective LDL apheresis. ${ }^{3}$

Different risk factors play important roles in the premature atherosclerosis in individuals with $\mathrm{hFH}$. Although an elevated LDL-C level is a major risk factor, it does not solely explain the incidence of early cardiovascular disease (CVD) in these subjects. ${ }^{4}$ Even other classic CVD risk factors, such as sex, smoking, hypertension, diabetes mellitus, low high-density lipoprotein (HDL-C) levels, and elevated lipoprotein (a) concentrations explain only $18.7 \%$ of the variability of the CVD occurrence in individuals with $\mathrm{hFH} .{ }^{4}$ This fact indicates the 
necessity to assess other risk factors that may participate in the development of accelerated atherosclerosis in this population. The hypothesis of this study was that severe periodontitis may be a risk factor for atherosclerosis in individuals with $\mathrm{hFH}$.

Periodontitis is a group of infections characterized by the progressive destruction of the tooth-supporting tissues. It has a multifactorial etiology that involves a complex interaction among specific bacteria, the host response, and risk factors. An association between periodontal diseases and atherosclerosis was suggested. . $^{5-9}$ Severe periodontitis may aggravate some subclinical atherosclerosis markers (such as intima-medial thickness [IMT]), hypertension, and atherogenic inflammatory markers (such as C-reactive protein [CRP] and white blood cell [WBC] counts)..$^{5,7,9-14}$

The present study investigates whether severe periodontitis affected clinical, metabolic and inflammatory parameters as well markers of subclinical vascular diseases, including pulse wave velocity (PWV) and carotid artery IMT, in individuals with $\mathrm{hFH}$.

\section{MATERIALS AND METHODS}

The project was approved by the Research Ethics Committee, Medical School Hospital, University of Sao Paulo, and informed consent was obtained from all participants. From 2005 to 2008, 81 consecutive patients with $\mathrm{hFH}$ who were followed at the Lipid Clinic of the Heart Institute, Medical School Hospital, University of Sao Paulo, were enrolled in the study. Two individuals were excluded because their data were incomplete. The remaining 79 individuals were analyzed (31 males and 48 females; age range: 17 to 80 years).

$\mathrm{hFH}$ was diagnosed by the United States Make Early Diagnosis to Prevent Early Death criteria. ${ }^{1}$ The exclusion criteria for the study were: 1 ) previous cardiovascular events, 2) diabetes mellitus, 3) thyroid diseases, 4) congestive heart failure, 5) pregnancy, 6) renal diseases, 7) any current inflammatory disease, 8 ) serum calcium disturbances, and 9) use of antibiotics and lipid lowering drugs in the 3 months and 6 weeks, respectively, before the study.

\section{Periodontal Evaluation}

Periodontal examinations were performed by a calibrated dental examiner (CLZV) according to the protocol described by Grossi et al. ${ }^{15}$ The clinical attachment loss (AL) was obtained indirectly from measurements of the probing depth (PD) and gingival recession. PDs and clinical ALs were measured using a manual probe. $\S$

Subjects with $\mathrm{hFH}$ were categorized into two groups: the severe periodontitis group (SPG) and the non-severe periodontitis group (NSPG). Individuals with severe periodontitis had $\geq 3$ sites, not on the same tooth, with clinical $\mathrm{AL} \geq 7 \mathrm{~mm}$ and $\geq 1$ interproximal sites with PDs $\geq 5 \mathrm{~mm}$. The NSPG included individuals who did not meet these conditions but had moderate periodontitis (i.e., $\geq 3$ sites with clinical $\mathrm{AL} \geq 5 \mathrm{~mm}$, not on the same tooth, and PDs $\geq 4 \mathrm{~mm}$ ), gingivitis, and periodontal health according to the criteria adapted from Bassani et al. ${ }^{16}$

In addition, the periodontal characteristics of both groups were further explored by analyzing medians and 95\% confidence intervals (CIs) for tooth loss, PDs $\geq 4$ and $\geq 5 \mathrm{~mm}$, and clinical ALs $\geq 6 \mathrm{~mm}$ for the SPG and NSPG.

\section{Clinical Evaluation and Blood Sample Analyses}

Subjects were queried about previous diseases. The body mass index (BMI) was calculated as weight in kilograms divided by height in square meters. ${ }^{2}$ Blood pressure was measured as previously recommended. ${ }^{17}$

Of a total of 79 subjects, 28 (35.4\%) subjects who were on previous statin therapy underwent a washout period of 6 weeks before evaluation as previously published. ${ }^{18}$ Blood samples were collected after a 12 -hour overnight fast. Sampling and blood pressure measurements were performed in the morning. The methods used in this study were previously described. ${ }^{19}$

Total cholesterol (TC), HDL-C, triglyceride, and glucose levels were determined according to standard laboratory methods. LDL-C levels were calculated by the Friedewald equation for triglyceride levels $<400 \mathrm{mg} / \mathrm{dL}$. Non-HDL-C levels were calculated by subtracting HDL-C levels from TC levels. High-sensitivity CRP levels were determined with immunonephelometry, and WBC counts were performed by using an automated method as previously described. ${ }^{19}$ The cholesterol-year score (CYS), which is a measurement of exposure to high cholesterol levels, was calculated by the age and TC product as previously described. ${ }^{19}$

\section{Evaluation of PWV, IMT, and Inflammatory Markers}

Methods for PWV and IMT were described elsewhere. ${ }^{19}$ In brief, IMT was measured longitudinally in the right common carotid artery and was analyzed by an automated edge-detection imaging pulsate ultrasonographic echo-tracking devicell with a probe of $7.5 \mathrm{MHz}$. To assess arterial stiffness, the carotidfemoral PWV was evaluated with a non-invasive device. "l Subclinical vascular disease and inflammatory markers such as IMT, PWV, CRP, and WBC counts were analyzed as continuous and categoric variables. An IMT $\geq 0.8 \mathrm{~mm}$, PWV $>12 \mathrm{~m} /$ second, CRP $>3.0 \mathrm{mg} / \mathrm{L}$, and WBC counts $>10,000$ cells $/ \mathrm{mm}^{3}$ were considered markers of severity of subclinical disease and inflammation.

\footnotetext{
§ PCP-86, Hu-Friedy, Chicago, IL.

Wall-Track System 2, PIE MEDICAL, Maastricht, The Netherlands.

If Complior apparatus, Colson, Paris, France.
} 


\section{Statistical Analyses}

Means, SDs, medians, and interquartile intervals were computed. Differences between the SPG and NSPG were evaluated using the unpaired $t$ test or MannWhitney $U$ test depending on the data distribution.

All data were categorized, and $\chi^{2}$ or Fisher exact tests were used to determine associations among all variables and periodontal disease categories. The variables that showed significant or marginally significant differences $(P \leq 0.2)$ between groups (SPG and NSPG) were tested in multivariate logistic regression analysis using a backward conditional regression method. The Hosmer-Lemeshow test was used to determine the adjustment level of the model and how much a group of independent variables could explain an outcome. The level for significance was set at $P \leq 0.05$.

A multiple correspondence analysis with canonical standardization was used to evaluate the geometric relationship, in the $\chi^{2}$ distance, among variables that showed significant or marginally significant differences $(P \leq 0.2)$ between groups in a multidimensional and multifactorial model.

Analyses were performed using a statistical software package.\#

\section{RESULTS}

A total of $41.8 \%(n=33)$ of individuals with hHF were within the SPG, and 58.2\% $(n=46)$ of individuals with hHF were within the NSPG. Clinical and laboratory characteristics of both groups are given in Table 1 . The groups did not differ regarding income, sex distribution, smoking habit, BMI, subfractions, and TC, CRP, and WBC levels $(P \geq 0.05)$. In the SPG, older age and higher diastolic blood pressure (DBP), CYS, triglyceride, glucose, TC, and HDL-C levels were observed $(P \leq 0.05)$. In contrast, there was no statistically significant difference among groups regarding systolic blood pressure $(P=0.88)$. The SPG also displayed higher values of PWV and IMT comparison with those of the NSPG $(P=0.03$ and $P=0.02$, respectively).

Periodontal characteristics of both groups are further described in Table 2. Percentages of PD $\geq 4$ and $\geq 5 \mathrm{~mm}$ and clinical $\mathrm{AL} \geq 6 \mathrm{~mm}$ were significantly higher for the SPG than for the NSPG $(P \leq 0.002)$.

Using multivariate analysis by logistic regression, the association between severe periodontitis and DBP (95\% CI; Hosmer-Lemeshow $P=0.031$ ) was confirmed after adjustment for traditional atherosclerotic risk factors, i.e., TC, age, smoking, HDL-C, BMI, and blood pressure (Table 3 ). Nevertheless, in the same analysis, there were no statistically significant associations between severe periodontal disease and a PWV $>12 \mathrm{~m} /$ second $(95 \% \mathrm{CI}$; Hosmer-Lemeshow $P=$ 0.512 ) or IMT $>0.8 \mathrm{~mm}$ (95\% Cl; Hosmer-Lemeshow $P=0.132)$.
As a representation of the multivariate analysis, Figure 1 shows that whereas only high DBPs were significantly associated with the SPG, normal DBP, PWV, IMT, and WBC counts were all closely associated with the NSPG. The positive values (severe periodontitis) were explained by the model in 55.9\% of the group, i.e., $55.9 \%$ of the SVG showed DBP, PWV, and IMT and normal WBC counts; all variables as a cluster. The negative values in the NSPG were explained in $74.5 \%$ of the group by the model, i.e., $74.5 \%$ of the NSPG showed normal values for DBP, PWV, and IMT and high WBC counts; all variables as a cluster.

\section{DISCUSSION}

In the multivariate analysis, a significant association was found between severe periodontitis and a higher DBP after adjusting for classic CHD risk factors. The data suggested that severe periodontitis, itself, may have contributed to the increased cardiovascular risk profile in this high-risk population. It is interesting to note that a direct relationship between the levels of subgingival periodontal bacteria and systolic blood pressure and DBP as well as hypertension prevalence has been recently described in subjects with no history of stroke or myocardial infarction. ${ }^{14}$ Infectious agents related to periodontal disease may affect DBP indirectly through the increase of inflammatory substances in the circulation and directly at the endothelial wall level.

In the present study, severe periodontitis is not associated with the PWV or IMT in the multivariate analysis. These results could be explained by the strong effects of other CVD risk factors presented in this special population, which could probably have overshadowed the effects of severe periodontitis. In contrast, the association between periodontitis and subclinical atherosclerosis was previously shown in other studies. ${ }^{5,7,9,11,20}$ In the bivariate analysis, we found that individuals with $\mathrm{hFH}$ in the SPG had higher values of PWV and IMT than did individuals with $\mathrm{hFH}$ in the NSPG. The different results observed in the multivariate analysis versus the bivariate analysis may have occurred because of sample size. Normal values of DBP, PWV, IMT, and WBC counts were more closely associated with the NSPG than the altered parameters were with the SPG. This result may imply that the specificity of the association between non-severe periodontitis and normal DBP, PWV, IMT, and WBC counts is more relevant compared to the association between severe periodontitis and the altered parameters.

Although previous studies demonstrated significantly higher TC and LDL-C levels and low HDL-C levels in individuals with severe periodontitis, ${ }^{21-24}$ we did not confirm this relationship. The most

\# SPSS, version 15.0, IBM, Chicago, IL. 
Table I.

\section{Clinical and Laboratory Characteristics of the Study Sample $(\mathrm{N}=79)$}

\begin{tabular}{|c|c|c|c|}
\hline Clinical and Laboratory Parameters & SPG $(n=33)$ & NSPG $(n=46)$ & $P$ \\
\hline Male (\%; mean [SD]) & $32(39)$ & $14(45.2)$ & 0.668 \\
\hline Age (years; mean [SD]) & $46.5(11.66)$ & $38.29(|4.7|)$ & 0.008 \\
\hline Smokers (\%) & 2 & 5 & 0.25 \\
\hline BMI $\left(\mathrm{kg} / \mathrm{m}^{2} ;\right.$ mean $\left.[\mathrm{SD}]\right)$ & $27.12(4.71)$ & $25.81(5.03)$ & 0.23 \\
\hline SBP (mm Hg; mean [SD]) & $120.64(17.25)$ & $120.12(15.06)$ & 0.88 \\
\hline DBP (mm Hg; mean [SD]) & $80.55(8.65)$ & $76.53(8.24)$ & 0.03 \\
\hline $\mathrm{TC}(\mathrm{mg} / \mathrm{dL} ;$ mean $[\mathrm{SD}])$ & $357.58(61.72)$ & $346.87(79.42)$ & 0.51 \\
\hline LDL-C (mg/dL; mean [SD]) & $279.29(60.98)$ & $265.42(73.11)$ & 0.36 \\
\hline $\mathrm{HDL}$ (mg/dL; mean [SD]) & $47.94(11.88)$ & $49.68(10.73)$ & 0.49 \\
\hline Non-HDL-C (mg/dL; mean [SD]) & $309.64(62.38)$ & $297.19(80.40)$ & 0.45 \\
\hline CYS (TC $\times$ age $[$ years]; mean [SD]) & $|6,865.4|(4,558.38)$ & 13,139.55 (6,228.56) & 0.004 \\
\hline $\begin{array}{l}\text { LDL-C/HDL-C (measure of risk } \\
\text { for CVD) }\end{array}$ & $6.22(2.40)$ & $5.66(2.31)$ & 0.29 \\
\hline $\begin{array}{l}\text { TG (mg/dL; median [interquartile } \\
\text { interval]) }\end{array}$ & $129(99.5$ to 210.75$)$ & 115 (9| to 157$)$ & 0.043 \\
\hline Glucose (mg/dL; mean [SD]) & $93.88(6.40)$ & $89.42(10.13)$ & 0.02 \\
\hline TG/HDL-C (measure of risk for CVD) & $3.53(2.19)$ & $2.73(1.46)$ & 0.05 \\
\hline $\begin{array}{l}\text { hs-CRP (mg/L; median [interquartile } \\
\text { interval]) }\end{array}$ & $2.57(1.30$ to 3.69$)$ & $1.22(0.47$ to 3.30$)$ & 0.06 \\
\hline WBC $10^{3} / \mathrm{mm}^{3} ;$ mean $\left.[\mathrm{SD}]\right)$ & $7,4 \mid 1.76(1,912.28)$ & $7,297.87(1,721.91)$ & 0.77 \\
\hline PWV (m/second; mean [SD]) & $9.67(1.65)$ & $8.99(1.16)$ & 0.03 \\
\hline IMT ( $\mu \mathrm{m} ;$ mean [SD]) & $710.91(164.64)$ & $633.12(157.94)$ & 0.03 \\
\hline
\end{tabular}

$\mathrm{TG}=$ triglycerides; $\mathrm{SBP}=$ systolic blood pressure, hs-CRP = high sensitivity CRP.

Table 2.

\section{Median (interquartile intervals) for Periodontal Disease Parameters}

\begin{tabular}{lcccc}
\hline Parameters & \multicolumn{1}{c}{ SPG } & NSP & $P$ \\
\hline TL $(\mathrm{n})$ & $9(4$ to 12.75$)$ & $6(4$ to II) & 0.653 \\
$\mathrm{PD} \geq 4 \mathrm{~mm}(\%)$ & $22.85(6.18$ to 31.17$)$ & $6.67(3.06$ to 19.5$)$ & 0.0023 \\
$\mathrm{PD} \geq 5 \mathrm{~mm}(\%)$ & $7.36(1.0$ । to $10.7 \mathrm{I})$ & $1.33(0$ to 2.98$)$ & $<0.000$ । \\
Clinical AL $\geq 6 \mathrm{~mm}(\%)$ & $14.29(1.19$ to 14.74$)$ & $1.19(0$ to 2.96$)$ & $<0.000$ । \\
\hline
\end{tabular}

$\mathrm{TL}=$ tooth loss. representative characteristic of patients with hFH were plasma LDL-C levels that were two-fold higher than normal, ${ }^{3}$ which is an important risk factor for CVD and might have obscured any possible effects of severe periodontitis on cholesterol fractions.

According to the multivariate analysis, the CYS and age were not associated with the severity of periodontitis. However, in the univariate analysis, the CYS and age were both significantly higher in the SPG compared to the NSPG, which may suggest an accumulated effect of periodontitis on the CYS over time.

Severe periodontitis was previously associated with an impairment of glycemic control and increased plasma triglycerides. ${ }^{25-28}$ In the multivariate analysis, we did not confirm these results after the adjustment for traditional atherosclerotic risk factors (i.e., TC, age, smoking, HDL-C, BMI, and blood pressure). Nevertheless, although the results from the univariate analysis represent less significance in the present study, the SPG showed higher glucose and triglyceride levels.

It was suggested that the effect of periodontitis on glucose metabolism may be apparent under conditions in which insulin action and/or secretion are partially impaired, including when there is high-fat diet exposition. ${ }^{28}$ In that study reference, the authors investigated the effects of high fat diet in the development of periodontitis. ${ }^{28}$ It is possible that in individuals with $\mathrm{hFH}$, the high levels of cholesterol have the same effects of the high-fat diet, thus explaining the higher levels of glucose in these subjects.

Indeed, chronic periodontitis shares common biologic 
Table 3.

\section{Multivariate Analysis by Logistic Regression of the Model That Explains the Relation Among Periodontal Disease and Blood Pressure, IMT, PWV, and WBCs in Patients With FH}

\begin{tabular}{lccc}
\hline $\begin{array}{l}\text { Subclinical } \\
\text { Atherosclerotic Marker }\end{array}$ & OR & Cl & $P$ \\
\hline DBP & 3.1 & I.I to 8.5 & 0.031 \\
PWV & 1.5 & 0.46 to 4.6 & 0.512 \\
IMT & 2.9 & 0.72 to 12.2 & 0.132 \\
WBC & 0.5 & 0.05 to 4.3 & 0.520 \\
\hline
\end{tabular}

Hosmer and Lemeshow: 0.960; constant: 0.007.

Representation of Multivariate Analysis by Logistic Regression

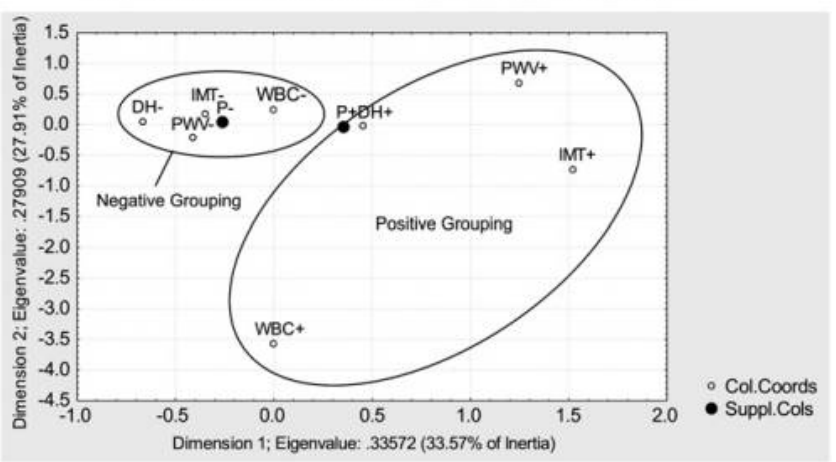

Figure I.

Representation of multivariate analysis by logistic regression. $\mathrm{DH}=$ diastolic hypertension (representing the DBP); IMT = intima-medial complex $>0.8 \mathrm{~mm} ; P=$ severe periodontitis; $P W V=P W V>12 \mathrm{~m} / \mathrm{second}$; $W B C=W B C$ count $>10,000$ cell/ $/ \mathrm{mm}^{3} ;$ Col. Coords (collaborative columns) = independent variables (i.e., DH, IMT, PWV, and WBC counts); Suppl. Cols (supplementary columns) = endpoint (severe periodontitis).

intersections with CVD, such as genetics, inflammation, and metabolic disturbances, which may underline the association between periodontal disease and poor glycemic control and high plasma triglyceride levels. ${ }^{25-27,29-32}$

Furthermore, the bidirectional relationship between periodontal disease and hyperglycemia, including type 2 diabetes mellitus, may support some aspects of theories about the development of metabolic syndrome and its association with periodontitis. ${ }^{25-32}$ Metabolic syndrome seems to result from sustained hyperglycemia, although the mechanism of contribution to cardiovascular pathogenesis from hyperglycemia is not well understood. ${ }^{32}$ The effects of hyperglycemia in the non-enzymatic glycation of proteins may stimulate macrophages to express cyto- kines, such as interleukin-6, and CRP, resulting in metabolic syndrome and atherosclerosis development. ${ }^{32}$ In this extensive discussion about the impact of periodontitis in the pathogenesis of atherosclerosis, several randomized trials ${ }^{33,34}$ successfully demonstrated that periodontal treatment decreased proinflammatory mediators associated with CVD pathogenesis and/or endothelial dysfunction.

Moreover, further studies may be capable of establishing a possible correlation between severe periodontitis and subclinical atherosclerosis, as well as identifying the specific effects of severe periodontitis in the aggravation of metabolic-syndrome markers in CVD patients, particularly in patients with $\mathrm{hFH}$.

\section{CONCLUSIONS}

Although the present study had limitations, especially the small sample size, it offered important information that can be used in future studies for sample-size calculation. In addition to the findings on the effects of periodontal disease in the development of atherosclerosis, this study disclosed that severe periodontitis may represent a risk factor for high DBP and may potentiate metabolic disturbances in the population with FH. To our knowledge, this finding signals a new path in the investigations of the mechanisms involved in the CVD development in the population with $\mathrm{FH}$, including the diagnosis of periodontal diseases. Further investigations should be made to identify the biologic mechanisms of the association between severe periodontitis and DBP in subjects with FH. Longitudinal studies are required to confirm the associations observed in the present study. If confirmed, these findings will have important clinical and public health implications, and health care strategies that target the diagnosis and treatment of periodontitis will be required in patients with $\mathrm{hFH}$.

\section{ACKNOWLEDGMENTS}

Dr. Vieira was supported by The National Council for Scientific and Technological Development, Brazilia, FD, Brazil (grant 140508/2006-6). The authors report no financial association with any products involved in this study.

\section{REFERENCES}

1. Williams RR, Hunt SC, Schumacher MC, et al. Diagnosing heterozygous familial hypercholesterolemia using new practical criteria validated by molecular genetics. Am J Cardiol 1993;72:171-176.

2. Santos RD, Meneghelo RS, Chacra AP, Martinez TL, Ramires JA, Carvalho JA. Detection of subclinical atherosclerosis by electron beam tomography in females with heterozygous familial hypercholesterolaemia. Heart 2004;90:92-94. 
3. Civeira F; International Panel on Management of Familial Hypercholesterolemia. Guidelines for the diagnosis and management of heterozygous familial hypercholesterolemia. Atherosclerosis 2004;173:55-68.

4. Jansen AC, Van Aalst-Cohen ES, Tanck MW, et al. The contribution of classical risk factors to cardiovascular disease in familial hypercholesterolaemia: Data in 2400 patients. J Intern Med 2004;256:482-490.

5. Beck JD, Elter JR, Heiss G, Couper D, Mauriello SM, Offenbacher S. Relationship of periodontal disease to carotid artery intima-media wall thickness: The atherosclerosis risk in communities (ARIC) study. Arterioscler Thromb Vasc Biol 2001;21:1816-1822.

6. Deshpande RG, Khan MB, Genco CA. Invasion of aortic and heart endothelial cells by Porphyromonas gingivalis. Infect Immun 1998;66:5337-5343.

7. Desvarieux M, Demmer RT, Rundek T, et al. Periodontal microbiota and carotid intima-media thickness: The Oral Infections and Vascular Disease Epidemiology Study (INVEST). Circulation 2005;111:576-582.

8. Dietrich T, Jimenez M, Krall Kaye EA, Vokonas PS, Garcia RI. Age-dependent associations between chronic periodontitis/edentulism and risk of coronary heart disease. Circulation 2008;117:1668-1674.

9. Deliargyris EN, Madianos PN, Kadoma W, et al. Periodontal disease in patients with acute myocardial infarction: Prevalence and contribution to elevated Creactive protein levels. Am Heart J 2004;147:10051009.

10. Loos BG, Craandijk J, Hoek FJ, Wertheim-van Dillen PM, Van der Velden U. Elevation of systemic markers related to cardiovascular diseases in the peripheral blood of periodontitis patients. J Periodontol 2000;71: 1528-1534.

11. Nakib SA, Pankow JS, Beck JD, et al. Periodontitis and coronary artery calcification: The Atherosclerosis Risk in Communities (ARIC) study. J Periodontol 2004;75: 505-510

12. Salzberg TN, Overstreet BT, Rogers JD, Califano JV, Best AM, Schenkein HA. C-reactive protein levels in patients with aggressive periodontitis. J Periodontol 2006;77:933-939.

13. Taguchi A, Sanada M, Suei Y, et al. Tooth loss is associated with an increased risk of hypertension in postmenopausal women. Hypertension 2004;43:12971300.

14. Desvarieux M, Demmer RT, Jacobs DR Jr., et al. Periodontal bacteria and hypertension: The oral infections and vascular disease epidemiology study (INVEST). J Hypertens 2010;28:1413-1421.

15. Grossi SG, Dunford RG, Ho A, Koch G, Machtei EE, Genco RJ. Sources of error for periodontal probing measurements. J Periodontal Res 1996;31:330-336.

16. Bassani DG, Olinto MT, Kreiger N. Periodontal disease and perinatal outcomes: A case-control study. J Clin Periodontol 2007;34:31-39.

17. Frohlich ED. Blood pressure measurement. Can $J$ Cardiol 1995;11(Suppl. H):35H-37H.

18. Kastelein JJ, Sager PT, de Groot E, Veltri E. Comparison of ezetimibe plus simvastatin versus simvastatin monotherapy on atherosclerosis progression in familial hypercholesterolemia. Design and rationale of the Ezetimibe and Simvastatin in Hypercholesterolemia Enhances Atherosclerosis Regression (ENHANCE) trial. Am Heart J 2005; 149:234-239.

19. Martinez LR, Miname MH, Bortolotto LA, et al. No correlation and low agreement of imaging and in- flammatory atherosclerosis markers in familial hypercholesterolemia. Atherosclerosis 2008;200:83-88.

20. Söder PO, Söder B, Nowak J, Jogestrand T. Early carotid atherosclerosis in subjects with periodontal diseases. Stroke 2005;36:1195-1200.

21. Katz J, Chaushu G, Sharabi Y. On the association between hypercholesterolemia, cardiovascular disease and severe periodontal disease. J Clin Periodontol 2001; 28:865-868.

22. Katz J, Flugelman MY, Goldberg A, Heft M. Association between periodontal pockets and elevated cholesterol and low density lipoprotein cholesterol levels. J Periodontol 2002;73:494-500.

23. Pussinen PJ, Jauhiainen $M$, Vilkuna-Rautiainen $T$, et al. Periodontitis decreases the antiatherogenic potency of high density lipoprotein. J Lipid Res 2004;45: 139-147.

24. Ramirez-Tortosa MC, Quiles JL, Battino M, et al. Periodontitis is associated with altered plasma fatty acids and cardiovascular risk markers. Nutr Metab Cardiovasc Dis 2010;20:133-139.

25. Taylor GW, Burt BA, Becker MP, Genco RJ, Shlossman M. Glycemic control and alveolar bone loss progression in type 2 diabetes. Ann Periodontol 1998;3:30-39.

26. Nibali L, D'Aiuto F, Griffiths G, Patel K, Suvan J, Tonetti MS. Severe periodontitis is associated with systemic inflammation and a dysmetabolic status: A case-control study. J Clin Periodontol 2007;34:931-937.

27. Lim LP, Tay FB, Sum CF, Thai AC. Relationship between markers of metabolic control and inflammation on severity of periodontal disease in patients with diabetes mellitus. J Clin Periodontol 2007;34:118-123.

28. Watanabe K, Petro BJ, Shlimon AE, Unterman TG. Effect of periodontitis on insulin resistance and the onset of type 2 diabetes mellitus in Zucker diabetic fatty rats. J Periodontol 2008;79:1208-1216.

29. D'Aiuto F, Sabbah W, Netuveli G, et al. Association of the metabolic syndrome with severe periodontitis in a large U.S. population-based survey. J Clin Endocrinol Metab 2008;93:3989-3994.

30. Noack B, Jachmann I, Roscher S, et al. Metabolic diseases and their possible link to risk indicators of periodontitis. J Periodontol 2000;71:898-903.

31. Khader Y, Khassawneh B, Obeidat B, et al. Periodontal status of patients with metabolic syndrome compared to those without metabolic syndrome. J Periodontol 2008; 79:2048-2053.

32. Janket SJ, Jones JA, Meurman JH, Baird AE, Van Dyke TE. Oral infection, hyperglycemia, and endothelial dysfunction. Oral Surg Oral Med Oral Pathol Oral Radiol Endod 2008;105:173-179.

33. D'Aiuto F, Parkar M, Nibali L, Suvan J, Lessem J, Tonetti MS. Periodontal infections cause changes in traditional and novel cardiovascular risk factors: Results from a randomized controlled clinical trial. Am Heart J 2006;151:977-984.

34. Tonetti MS, D'Aiuto F, Nibali L, et al. Treatment of periodontitis and endothelial function. N Engl J Med 2007;356:911-920.

Correspondence: Dr. Bruno Caramelli, Heart Institute, Medical School, University of São Paulo, Av. Dr. Eneas Carvalho de Aguiar 44, São Paulo, SP, Brazil. Fax: 55-11 30695535; e-mail: bcaramel@usp.br.

Submitted August 9, 2010; accepted for publication October 3, 2010. 